\title{
Lesões oculares por Processionária (Thaumetopoea pityocampa Schiff): relatos de casos
}

\author{
Processionary (Thaumetopoea pityocampa Schiff) induced ocular lesions: case reports
}

\author{
Francisco Eduardo da Silva Trincão ${ }^{1}$, Ana Filipa Duarte ${ }^{1}$, Ana Aires Magriço ${ }^{1}$, Vítor Santos Maduro ${ }^{1}$, Pedro Alves Albergaria Candelária ${ }^{1}$
}

\section{RESUMO}

A Processionária (Thaumetopoea pityocampaSchiff), vulgarmente conhecida como "lagarta do pinheiro"é um inseto dos pinheiros e cedros, endêmico em meios rurais mas também em meios urbanos. A toxicidade ocular, rara nas últimas décadas pelo desenvolvimento de métodos de erradicação eficazes, é provocada pelos seus pelos e prevê-se mais frequente com o recrudescimento deste inseto. Revemos a epidemiologia da Processionária e as suas lesões oculares a partir de 3 casos clínicos. Caso 1: Doente de 64 anos recorre ao Serviço de Urgência (SU) com olho direito vermelho e sensação de corpo estranho após prática de jardinagem. A observação revela AVODc: 0,5, erosão epitelial, presença de um filamento no estroma corneano profundo, flare $(++)$ e Tyndall $(+++)$. Caso 2: Doente de 28 anos, recorre ao SU por dor intensa no olho direito acompanhada de hiperemia após contato com lagarta. Apresenta AVODc: 0,6 e Tyndall (+++) com presença de múltiplos filamentos (mais de 20) a diferentes profundidades da córnea. Caso 3: Doente de 26 anos, recorre ao SU por sensação de corpo estranho e lacrimejamento constante no olho direito, após realizar exercícios militares num parque urbano. Apresenta AVODc: 0,3, múltiplas erosões epiteliais puntiformes na metade nasal da córnea que recobriam filamentos de cor laranja e Tyndall (+). Foi instituída terapêutica com corticoide tópico e vigilância sintomática a cada um dos casos. A patologia ocular por Processionária decorre da toxicidade dos seus pelos, cuja migração ocorre preponderantemente no sentido intraocular. Inclui por isso lesões precoces (conjuntivite, queratite e uveíte) e tardias (catarata, pars planite, vitreíte e retinite). Os casos apresentados possuíam lesões iniciais, tendo recuperado totalmente do quadro inflamatório após seis meses mas mantendo os pelos inativos no estroma corneano. A gravidade destes casos prende-se à possibilidade de migração intraocular, que pode ocorrer anos após o episódio inicial, obrigando a uma vigilância ao longo da vida.

Conclusão: O recrudescimento da Processionária, tanto em meios rurais como urbanos, justifica o conhecimento das lesões oculares que pode causar e o seu tratamento.

Descritores: Traumatismos oculares/etiologia; Lepidópteros; Toxicidade; Relatos de casos

\begin{abstract}
The Processionary (Thaumetopoea pityocampa Schiff), is an endemic insect of pine and cedar trees, existing both in rural and urban areas. Ocular toxicity, once rare due to the efficacy of eradication methods, is caused by the insects' hair and is expected to be more frequent with its recrudescence. We report two clinical cases and review the epidemiology of Processionary and its eye injuries. Case 1: a 64 year-old patient complained of red eye and foreign body sensation after being gardening. She had a BCVA of 0.5 and biomicroscopy revealed an epithelial erosion, flare $(++)$, Tyndall $(+++)$ and the presence of a setae in the deep corneal stroma. Case 2: a 28 year-old patient complained of severe pain in the right eye and hyperemia after having had contact with a caterpillar. He presented with a BCVA of 0.6, Tyndall (+++) and multiple filaments (over 20) at different depths in the cornea. Case 3: a 23 year-old patient refers to the ER with foreign body sensation and constant lacrimation in the right eye after having been completing military exercises in an urban park. He presented a BCVA of 0.3 , multiple epithelial erosions in the nasal half of the cornea that covered multiple orange strands and an anterior chamber reaction (Tyndall +). Therapy was initiated with topical steroids and symptomatic surveillance. Ocular toxicity due to Processionary hairs, whose movement occurs preferentially towards the posterior pole, includes early signs (conjunctivitis, keratitis, and uveitis) and late signs (cataract, pars planitis, vitritis, and retinitis). The 3 cases presented had early lesions, having fully recovered from the inflammatory condition after 6 months. However, inactive setae were still visible in the corneal stroma. Intraocular migration, which can occur years after the initial episode, is possible and might be severe, thus requiring a life-long surveillance.

Conclusion: Processionary recrudescence has been observed, both in rural and urban areas. It is therefore imperative to be familiar with eye injuries caused by the insect and its treatment.
\end{abstract}

Keywords: Eye injuries/etiology; Lepidoptera; Thaumetopoea; Toxicity; Case reports

\section{INTRODUÇÃO}

A Thaumetopoea pityocampa (Schiff), vulgarmente conhecida como "lagarta do pinheiro" é um inseto dos pinheiros e cedros, endêmico em meios rurais mas também em meios urbanos em Portugal. É frequentemente apelidada de Processionária por movimentar-se num grupo linear de lagartas sucessivas, como que em procissão, que atinge frequentemente dezenas de metros (Figuras 1 e 2). Além dos danos florestais que provoca, é tóxica para o homem e outros animais devido às características urticantes dos seus pelos. É responsável por toxicidade ocular, cutânea - com rash e prurido - e respiratória.

O ciclo biológico da Processionária completa-se geralmente num ano, distinguindo-se uma fase aérea (na copa dos pinheiros) e outra subterrânea (no solo), sendo esta de maior risco infeccioso. Assistindo-se nos últimos anos a francas alterações climáticas (invernos secos e pluviosidade reduzida), com as quais o grau de desenvolvimento da Processionária está intimamente relacionado, têm sido mais precoces as descidas das lagartas para o solo (final de outono - início de inverno) e consequentemente mais frequentes os casos de contato com humanos. Infestações por esta lagarta desfolhadora e casos de toxicidade humana foram recentemente verificadas em locais onde espécies de pinheiros e cedros são endêmicos (particularmente nos países do sul da Europa) $)^{(1,2)}$.

Apresentamos três casos clínicos demonstrativos da toxicidade ocular por este inseto.

\section{CASOS CLÍNICOS}

Caso 1: Doente de 64 anos de idade, sexo feminino, recorre ao SU por olho direito vermelho e sensação de corpo estranho. Referia que estes sintomas teriam tido início após realizar limpeza do quin- 
tal, onde se encontravam"várias lagartas em fila" (sic). A observação revelava uma melhor acuidade visual corrigida (MAVC): OD - 0,5, OE - 0,8. Na biomicroscopia salientava-se uma erosão epitelial única, paracentral inferior e era possível verificar, em grande ampliação, a presença de 1 filamento de coloração alaranjada no estroma corneano profundo. Apresentava câmara anterior formada, flare $(++)$ e Tyndall (+++), não sendo visíveis filamentos em suspensão, sobre a íris ou sobre o cristalino opalescente (catarata ODE N03NC3 - LOCS III). Tinha pressões intraoculares: OD - $14 \mathrm{mmHg}, \mathrm{OE}-16 \mathrm{mmHg}$, não apresentando alterações na fundoscopia.

Caso 2: Doente de 28 anos de idade, sexo masculino, recorre ao SU por dor intensa no olho direito acompanhada de hiperemia conjuntival. Referia contato ocular direto com lagarta, ao realizar limpeza florestal. Apresentava MAVC - OD: 0,6 e OE: 1,0 e a presença de múltiplas erosões puntiformes corneanas que correspondiam aos pontos de entrada de filamentos alaranjados (quantificados em mais de 20) a diferentes profundidades da córnea, alguns atravessando a Descemet (Figura 3). Não eram visíveis corpos estranhos intraoculares mas apresentava Tyndall $(+++)$.

Caso 3: Doente de 26 anos, recorre ao SU por sensação de corpo estranho e lacrimejamento constante no olho direito, após realizar

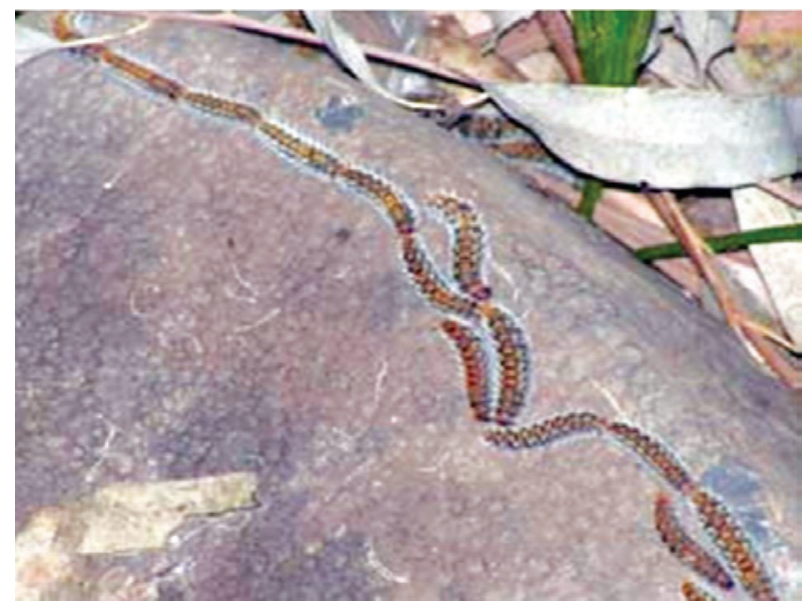

Figura 1. Fileira de Processionárias.

Fonte: Departamento do Ambiente e Espaços Verdes da Câmara Municipal de Lisboa (reproduzido com autorização).

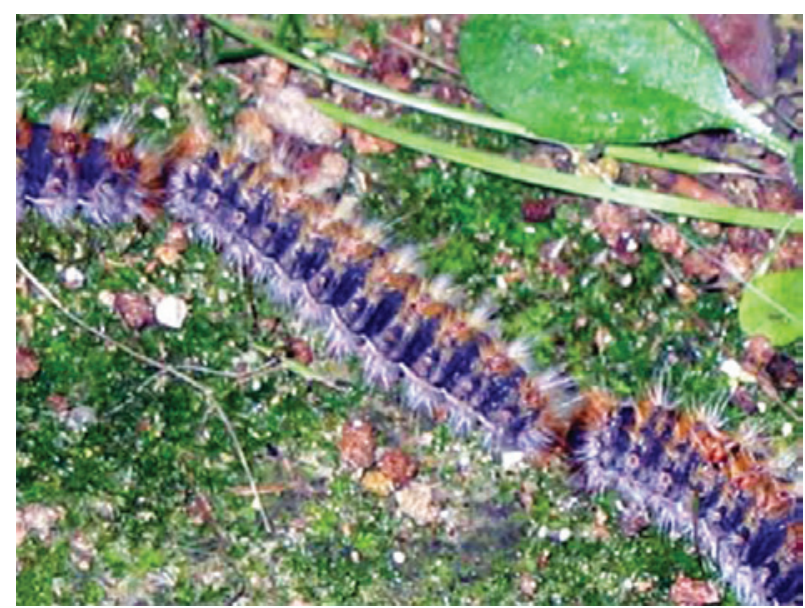

Figura 2. Processionária.

Fonte: Departamento do Ambiente e Espaços Verdes da Câmara Municipal de Lisboa (reproduzido com autorização). exercícios militares num parque urbano. Apresentava AVODc: 0,3 e múltiplas erosões epiteliais puntiformes na metade nasal da córnea que recobriam filamentos de cor laranja (Figura 4). A câmara anterior estava formada, com Tyndall (+) e sem filamentos observáveis. Também não foram observadas alterações no cristalino, vítreo ou fundo ocular.

Nos três casos apresentados, foi feito o diagnóstico presuntivo de toxicidade por Processionária e iniciada terapêutica com colírios de dexametasona (4 id), ofloxacina (4 id) e tropicamida (3 id), cuja dose foi sendo progressivamente diminuída. Em todos, verificou-se franca melhoria dos sintomas, acuidades visuais finais de 1,0 ao final de um mês e ausência de reação de câmara anterior. Contudo, o caso 2 manteve terapêutica com fluormetolona durante quatro meses adicionais por não tolerar a ausência de corticoterapia (lacrimejamento e ardor constantes).

De salientar que a presença dos filamentos na córnea se manteve até à data, tendo estes tomado uma aparência translúcida.

\section{COMENTÁRIOS}

O diagnóstico clínico de toxicidade ocular a Processionária exige um elevado grau de suspeição e uma observação minuciosa. Embora se manifeste habitualmente como queratite ou queratoconjuntivite (e em mais de 95\% destes casos com uveíte anterior aguda (3), são descritas outras localizações, na origem da classificação de Cadera et al. ${ }^{(4)}$ :

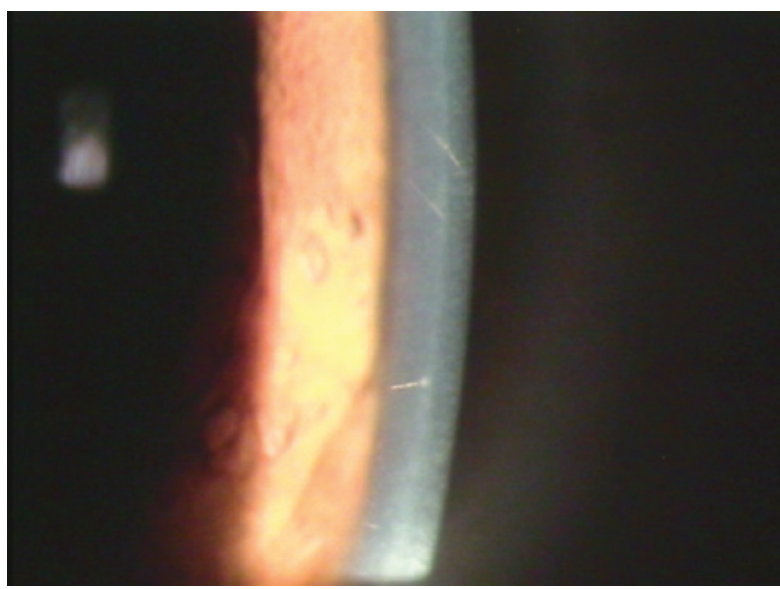

Figura 3. Pelos de Processionária em diferentes profundidades do estroma.
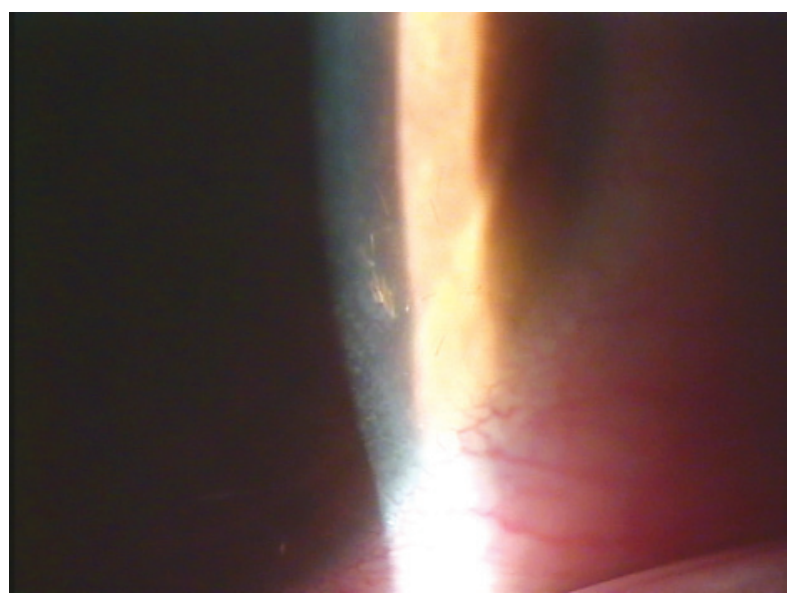

Figura 4. Erosão epitelial e pelos de Processionária subjacentes. 
Tipo 1 - Reação tóxica aguda com quemose, inflamação, epífora e sensação de corpo estranho.

Tipo 2 - Queratoconjuntivite crônica mecânica. Pelos na conjunctiva palpebral/bulbar com erosões corneanas correspondentes.

Tipo 3 - Granulomas subconjuntivais.

Tipo 4 - Irite e presença de filamentos na câmara anterior (e/ou cristalino)

Tipo 5 - Vitreíte e/ou retinite.

A resposta inflamatória ocular é induzida por duas características fundamentais do pelo da Processionária.

A primeira - mecânica - deve-se à sua forma em arpão, recoberto por uma substância viscosa, que favorece a progressão num único sentido(5). Esta, invariavelmente anteroposterior, não é espontânea mas antes favorecida pelos movimentos oculares, pulso, Valsalva e "rubbing", sendo por isso fundamental alertar o doente para evitar estas manobras ${ }^{(6-8)}$.

A segunda - tóxica - associada à taumatopoietina, proteína secretada pela glândula pilosa acessória da Thaumatopoea, e que induz uma forte resposta imunológica quando o pelo agride a Descemet ${ }^{(9)}$.

Estas particularidades morfológicas tornam as formas de atingimento corneano de abordagem delicada. A tentativa de remoção mecânica do pelo pode propiciara sua migração intraocular, pelo que deve ser extremamente cuidadosa ou mesmo evitada e se possível substituída apenas pela lavagem copiosa da superfície ocular ${ }^{(10)}$.

A presença de Tyndall torna fortemente provável o contato do pelo com o humor aquoso ${ }^{(11)}$. A sua frequente integração no estroma iridiano, ocultação no segmento posterior ou eliminação trabecular tornam-no raramente visível, o que pudemos comprovar no nosso trabalho (apenas no caso 2 conseguimos demonstrar este contato).

Embora estejam descritos casos de irite, granulomas conjuntivais ou iridianos, vitreíte e endoftalmite ${ }^{(10,12,13)}$, os nossos doentes tiveram uma excelente evolução clínica até a data, com resolução completa nos primeiros meses. $\mathrm{O}$ risco de migração posterior do pelo obriga contudo a uma vigilância periódica durante vários anos. A presença de cristalino (e particularmente a sua cápsula anterior) poderá representar neste campo uma vantagem, ao proporcionar uma possível barreira à progressão do pelo da câmara anterior para o vítreo ${ }^{(7,14,15)}$. Neste contexto, poder-se-ia especular que um período janela até à facectomia permitiria a impactação de pelos que estivessem presentes na câmara anterior.

Sridhar et al. propõem um algoritmo terapêutico ${ }^{(16)}$ de acordo com a classificação de Cadera et al. (4) $^{(4)}$

Tipos 1 ou 2: irrigação seguida de remoção meticulosa dos pelos. Antibioterapia e corticoterapia tópicas.

Tipo 3: excisão cirúrgica dos nódulos.

Tipo 4: corticoterapia tópica, iridectomia nodular ou remoção direta dos pelos.

Tipo 5: corticoterapia sistémica com vitrectomia e remoção dos pelos em casos refratários.
A evolução natural das lesões descritas é a da formação progressiva de nódulos em torno dos pelos, que correspondem a agregados de linfócitos, macrófagos e células epitelioides, rodeados duma cápsula fibrosa. Macroscopicamente, verifica-se ao longo do tempo um progressivo aclaramento do pelo no estroma que toma frequentemente um aspeto translúcido e que corresponde à perda da toxina e sua consequente inativação. Não é contudo claro se após esta fase o acompanhamento deva ser mantido apertado.

\section{CONCLUSÃO}

A toxicidade ocular por Processionária é cada vez mais frequente nos países mediterrâneos e a gravidade decorre da possível penetração intraocular dos seus pelos.

Numa forte suspeita de contato, o diagnóstico deve ser confirmado por uma observação cuidadosa da córnea que revela a presença de filamentos na sua espessura. O recrudescimento deste inseto obriga o conhecimento das lesões oculares que pode provocar e seu tratamento.

\section{REFERÊNCIAS}

1. Ministério da Agricultura, Desenvolvimento Rural e das Pescas. Direção Geral das Florestas. Processionária do Pinheiro. Portugal: Ministério da Agricultura, do Desenvolvimento Rural e das Pescas; 2004.

2. Lisboa. Câmara Municipal. Direcção Municipal de Ambiente Urbano; Departamento do Ambiente e Espaços Verdes. Divisão de Matas. Lagartas Processionárias nos Parques de Lisboa. Lisboa: Câmara Municipal; 2009.

3. Bishop JW, Morton MR. Caterpillar-hair keratoconjunctivitis. Am J Ophthalmol. 1967; 64(4):778-9.

4. Cadera W, Pachtman MA, Fountain JA, Ellis FD, Wilson FM $2^{\text {nd }}$. Ocular lesions caused by caterpillar hairs (ophthalmia nodosa). Can J Ophthalmol. 1984;19(1):40-4.

5. D'Hermies F, Parent de Curzon H, Mathieu L, Furia M, Campinchi R. [Chorioretinopathy caused by migration of caterpillar hairs. A propos of 2 cases]. J Fr Ophtalmol. 1985;8(6-7):471-8. French.

6. Shibui H, Kawashima H, Kamata K, Sasaki H, Inoda S, Shimizu H. Vitrectomy for caterpillar seta-induced endophthalmitis. Arch Ophthalmol.1997;115(4):555-6.

7. Gundersen T, Heath P, Garron LK. Ophthalmia nodosa. Trans Am Ophthalmol Soc. 1950 48:151-69.

8. Villard H, Dejean CH. L'ophtalmie des chenilles. Arch Ophthalmol. 1934;51:719-45.

9. Lamy M, Pastureaud MH, Novak F, Ducombs G, Vincendeau P, Maleville J, Texier L. Thaumetopoein: an urticating protein from the hairs and integument of the pine processionary caterpillar (Thaumetopoea pityocampa Schiff). Toxicon. 1986;24(4):347-56.

10. Fraser SG, Dowd TC, Bosanquet RC. Intraocular caterpillar hairs (setae): clinical course and management. Eye (Lond). 1994;8(Pt 5):596-8.

11. Conrath J, Hadjadj E, Balansard B, Ridings B. Caterpillar setae-induced acute anterior uveitis: a case report. Am J Ophthalmol. 2000;130(6):841-3.

12. Steele C, Lucas DR, Ridgway AE. Endophthalmitis due to caterpillar setae: surgical removal and electron microscopic appearances of the setae. Br J Ophthalmol. 1984; 68(4):284-8.

13. Corkey JA. Ophthalmia nodosa due to caterpillar hairs. Br J Ophthalmol. 1955;39(5):301-6

14. Rishi P, Agarwal M, Mahajan S, Rishi E. Management of intralenticular caterpillar setae. Indian J Ophthalmol. 2008;56(5):437-8.

15. Ascher KW. Mechanism of locomotion observed on caterpillar hairs. Br J Ophthalmol. 1968;52(2):210.

16. Sridhar MS, Ramakrishnan M. Ocular lesions caused by caterpillar hairs. Eye (Lond) 2004;18(5):540-3. 